Sociohistórica

ISSN: $1852-1606$

publicaciones@fahce.unlp.edu.ar

Universidad Nacional de La Plata

Argentina

\title{
De la calle al palacio. La resolución parlamentaria del conflicto por el Impuesto a las Ganancias (2016)
}

Corral, Damian; Wyczykier, Gabriela

De la calle al palacio. La resolución parlamentaria del conflicto por el Impuesto a las Ganancias (2016)

Sociohistórica, núm. 46, 2020

Universidad Nacional de La Plata, Argentina

DOI: https://doi.org/10.24215/18521606e111

Atribución no comercial compartir igual (CC BY-NC-SA) 4.0 
Artículos

\section{De la calle al palacio. La resolución parlamentaria del conflicto por el Impuesto a las Ganancias (2016)}

From the street to the palace. The parliamentary resolution of the conflict over the Income Tax (2016)

Damian Corral

DOI: https://doi.org/10.24215/18521606e111

Universidad Nacional de General Sarmiento / CONICET,

Argentina

corral.damian@gmail.com

Recepción: 19 Julio 2019

Gabriela Wyczykier

Aprobación: 12 Marzo 2020

Universidad Nacional de General Sarmiento / CONICET,

Argentina

gwyczykier@yahoo.com

Recepción: 19 Julio 2019

Aprobación: 12 Marzo 2020

\section{RESUMEN:}

El artículo analiza el conflicto sociopolítico en torno del Impuesto a las Ganancias, ocurrido durante el segundo mandato de Cristina F. de Kirchner y el primer año de gestión de la Alianza Cambiemos, prestando particular atención a la última fase de la disputa acontecida enel 2016, donde el reclamo se trasladó “de la calle al palacio”, luego de una sucesión deacciones colectivas encaradas por una parte del sindicalismo.

Se abordará este proceso a partir detres dimensiones centrales: la relación de los sindicatos con el gobierno de Mauricio Macri, la reconfiguración de las alianzas parlamentarias y las modificaciones realizadas en el tributo en el recinto legislativo. Los argumentos trabajados surgen de una investigación cualitativa y de orientación sociopolítica.

El texto reflexiona sobre un conflicto de presencia sustantiva en la agenda política y sindical, al tiempo que examina la reconfiguración de alianzas parlamentarias en la era Cambiemos

Palabras Clave: Conflicto sociopolítico, Impuesto a las Ganancias, Sindicalismo, Actores parlamentarios, Gobierno.

\section{Abstract:}

The article analyzes the sociopolitical conflict around the Income Tax, that occurred during the second term of Cristina F. de Kirchner and the first year of management of the Alianza Cambiemos, paying particular attention to the last phase of the dispute that took place in 2016, where the claim was moved "from the street to the palace", after a succession of collective actions addressed by a part of the trade union movement.

This process will be approached from three central dimensions: the relationship of the unions with the government of Mauricio Macri, the reconfiguration of the parliamentary alliances and the modifications made in the tribute in the legislative enclosure. The arguments worked arise from a qualitative research and sociopolitical orientation.

The text reflects on a conflict of substantive presence in the political and trade union agenda, while examining the reconfiguration of parliamentary alliances in the era Cambiemos

KEYWORDS: Sociopolitical Conflict, Income Tax, Unionism, Parliamentary Actors, Government.

\section{INTRODUCCIÓN}

El enfrentamiento en torno del Impuesto a las Ganancias (IG) en la Argentina impregnó la agenda de reivindicaciones de una parte del sindicalismo de mayor peso confrontativo desde el 2012 hasta el 2016, en particular el que refiere a la cuarta categoría, que impacta sobre los salarios e ingresos de los trabajadores. La demanda por la derogación y/o modificación del gravamen se expresó en los cinco paros generales que encaró la Confederación General del Trabajo -CGT-Azopardo, ${ }^{1}$ la Azul y Blanca, ${ }^{2}$ la Central de los Trabajadores 
de la Argentina -CTA-Autónoma, ${ }^{3}$ organizaciones partidarias, gremiales, y en una sucesión de medidas de fuerza sectoriales desarrolladas durante los últimos tres años del gobierno de Cristina F. de Kirchner. ${ }^{4}$ Al mismo tiempo, el conflicto estuvo presente en las campañas de los principales candidatos a disputar la sucesión presidencial, y dio lugar a la gestación de una serie de proyectos parlamentarios promovidos por distintas fuerzas políticas, que, sin embargo, no lograron tratamiento legislativo en aquel período de gobierno.

Así, una problemática tributaria que afectaba aproximadamente al $10 \%$ de la masa de asalariados con mejores remuneraciones relativas trasmutó en un conflicto sociopolítico que signó las relaciones entre el sindicalismo y el kirchnerismo. Si bien este tipo de tributo surgió en el país en el año 1933 y en distintas etapas históricas se advirtieron disconformidades de algunos grupos sociales con referencia a las características de su alcance, los atributos del conflicto por el IG resultan peculiares, así como lo ha sido la manera en que se involucraron en la contienda los sectores del trabajo, del gobierno, y parte de la dirigencia política opositora. Efectivamente, este conflicto interpeló a diversos actores partidarios que buscaron aprovechar la situación para obtener réditos electorales, como fue el caso Sergio Massa y el de Mauricio Macri, candidatos presidenciales por el Frente Renovador y de la coalición Cambiemos, respectivamente.

El tratamiento político del conflicto por el IG se reconfiguró a partir del triunfo de Mauricio Macri en las elecciones presidenciales de noviembre de 2015 y del arribo al gobierno nacional de una nueva alianza de partidos (PRO, Unión Cívica Radical, Coalición Cívica).En este nuevo contexto, influyeron en la orientación respecto del gravamen las transformaciones económicas, la relación con los actores sindicales y la nueva dinámica legislativa.

Ciertamente, los lineamientos rectores del programa económico implementado por el presidente Macri resultaron sustancialmente diferentes de los impulsados por el gobierno anterior. Uno de los contrastes más notorios se advirtió en la política de empleo, objetivo prioritario durante la etapa kirchnerista, que comenzó a desarticularse en la gestión Cambiemos. Si bien durante el 2016 el empleo público creció 0,9 \% en relación al año anterior, ${ }^{5}$ distinta fue la performance del empleo privado. Durante ese año se perdieron 44.465 puestos de trabajo, mientras que la subocupación ascendió entre 2015 y 2016 al 10, 6\%. Por su parte, en el marco de un considerable aumento de precios en los bienes de consumo y servicios domésticos, ${ }^{6}$ el poder adquisitivo de los salarios perdió un $6 \%$ en promedio para los trabajadores registrados.

En este contexto, se reconfiguraron las demandas del movimiento sindical que, luego de haber centrado su acción colectiva en el reclamo por modificar el IG para la cuarta categoría durante el segundo mandato de Cristina Fernández de Kirchner, comenzó a priorizar la defensa de puestos laborales amenazados. Estos cambios influyeron en la modificación de la estrategia de ambas CGT, orientada hacia la reunificación y al ejercicio del diálogo y la negociación con el gobierno. En este marco, en diciembre de 2016 empezaron a ser tratadas modificaciones al IG en el parlamento, con intensos debates y articulación de alianzas legislativas para lograr modificar una serie de aspectos que caracterizaban al gravamen.

Así, a la vez que el tratamiento legislativo del IG permite dar cuenta del modo en que el conflicto logró una resolución -aunque parcial- de la problemática, revela al mismo tiempo cómo se fue configurando el entramado político en el parlamento que promovió el flamante presidentepara lograr gobernabilidad en sus primeros tiempos del mandato.

En este artículo proponemos analizar el conflicto sociopolítico por el IG prestando particular atención a la última fase de la disputa en el 2016. Para ello abordaremos tres dimensiones centrales: la relación de los sindicatos con el gobierno de Macri, la configuración de las alianzas parlamentarias, y las modificaciones realizadas en el gravamen. En primer lugar, enunciaremos brevemente algunos hitos de la confrontación por el IG que tuvieron lugar en momentos previos y que se advierten como el preludio de la modificación regulatoria del 2016, para luego concentrar la exposición en la última fase de la contienda, que denominamos "de la calle al palacio". 
Los enunciados vertidos en estas páginas surgen de una investigación cualitativa para la que se realizaron entrevistas en profundidad a informantes calificados, ${ }^{7}$ se sistematizó información presentada en medios de difusión masiva, se realizó una observación de informes técnicos y un estudio de documentos taquigráficos de las sesiones parlamentarias. ${ }^{8}$

El análisis de la información llevado adelante a partir de esta perspectiva metodológica nos ha permitido considerar valoraciones, acciones y estrategias que impulsaron sus protagonistas en un entramado de poder y relaciones de dominación asimétrica. Como bien plantea O Donnell (1978), estas apreciaciones contribuyen a comprender cómo se configuran y reconfiguran en el plano político-ideológico las posiciones de los distintos actores sociales, dominantes y subalternos, en relación con su posición estructural y su modalidad de organización corporativa.

\section{TOMAR LA CALLE: LA CONFRONTACIÓN POR EL IMPUESTO A LAS GANANCIAS EN EL KIRCHNERISMO}

El conflicto en torno del IG se configuró como uno de carácter sociopolítico. Esto implica, como sostiene Tilly (1998), que un grupo persigue reivindicaciones colectivas a través de actos visibles en el espacio público. Entre los actores involucrados, una de las partes afectada e interpelada por las demandas es el gobierno. En consecuencia, un conflicto político abarca distintos procesos contenciosos y repertorios de acción.

Los conflictos políticos encarnan por tanto enfrentamientos que acontecen entre actores colectivos que mantienen distintas percepciones, valoraciones, y perspectivas sobre un tema y una problemática. Los repertorios de acción y la expresión de las reivindicaciones y las demandas se suceden muchas veces alternando períodos de abierta confrontación y enfrentamiento en el espacio público, con momentos en los cuales las demandas se producen de manera enunciativa y deliberativa.

En relación al conflicto sociopolítico por el IG, puede tomarse como uno de sus puntos iniciales el primer paro general del 20 de noviembre de 2012, liderado por el dirigente camionero Hugo Moyano de la CGT Azopardo, Luis Barrionuevo de la Azul y Blanca y Pablo Micheli de la CTA-Autónoma. Entre las demandas de los gremios convocantes, la eliminación del gravamen hacía su aparición como un reclamo de jerarquía que hubo de impregnar la agenda sindical de las agrupaciones que mostraron sus disidencias con el segundo mandato de Cristina F. de Kirchner en los años subsiguientes. La disconformidad, que habían advertido de modo enunciativo dirigentes gremiales en años anteriores (a partir del 2007 comenzaron a plantearle sus disidencias al gobierno), se trasladó entonces como reclamo a la calle.

Esta medida de fuerza tuvo como antecedente la ruptura que se había consumado entre el gobierno y Hugo Moyano, cuando la división entre la CGT “Azopardo” “ “Alsina” -la primera bajo el liderazgo del líder camionero y la segunda bajo el del dirigente metalúrgico Antonio Caló- cristalizó ese proceso. Dicho quiebre institucional fue el resultado de tensiones que ya se habían preanunciado desde el 2009 y 2010 en adelante, relacionadas con demandas corporativas no atendidas por el gobierno nacional, pero también con respecto a las propias pujas en el interior de la confederación por las distintas orientaciones políticas y gremiales comprendidas en ella.

Entre las causas de la ruptura puede mencionarse el reclamo de 2010 por la regulación de un proyecto alentado por Hugo Moyano y el abogado Héctor Recalde para lograr que, en un principio, los trabajadores de grandes empresas tuvieran una participación en las ganancias. También pueden mencionarse los enfrentamientos por la definición de las listas electorales del Frente Para la Victoria, el avance de la juventud de La Cámpora en la estructura de gobierno, los cambios en la dinámica económica y las dificultades de sostener el crecimiento con redistribución como ocurriera años anteriores. Además, incidió la conformación de un espacio sindical opositor en la CGT, que se propuso disputar cargos al grupo de Moyano en las elecciones de 2012, y la instalación de la figura de Caló (ligada al sector industrial revitalizado durante la 
década) para presidir el secretariado general de la CGT -candidatura apoyada por funcionarios del gobierno(Natalucci y Morris, 2016; Marticorena, 2015; Varela, 2014; Schipani, 2012).

Con la división de la CGT, junto a la CGT Azul y Blanca presidida por Luis Barrionuevo, y a las dos centrales de trabajadores argentinos (CTA de los Argentinos y CTA Autónoma), la representación de los trabajadores quedaba expresada entonces en cinco agrupaciones sindicales de tercer grado al comenzar el conflicto por el IG.

Luego de esta primera medida de fuerza se realizaron dos paros generales en 2014 ( 10 de abril y 28 de agosto), y otros dos en 2015 ( 31 de marzo y 9 de junio), en los cuales la demanda por el IG tuvo protagonismo. Las mismas se produjeron en un clima de descontento sindical respecto de acciones del gobierno, pero también en un contexto de campaña electoral para las elecciones presidenciales. Junto a los paros generales se desarrollaron en ese período una serie de medidas de fuerza sectoriales de parte de algunos gremios (bancarios, gremios del transporte, algunas delegaciones de Luz y Fuerza) para exigir al gobierno la derogación o modificación del IG.

La consolidación del conflicto sociopolítico por el IG estuvo entonces signada por un conjunto de problemáticas que lo singularizaron: la relación de Hugo Moyano con la presidenta a partir del 2011, y las divisiones y disputas en el interior de las organizaciones sindicales. Por tanto, la nueva ruptura de la CGT del 2012 -cuya última unificación databa de 2004-, la división de la CTA en 2010, ${ }^{9}$ la distancia que fueron adoptando distintos gremios con algunas políticas de gobierno, ${ }^{10}$ y el apoyo de dirigentes partidarios de la oposición al conflicto -en el afán de concitar adhesiones propias, así como de partidos de izquierda que consideraban que la clase trabajadora no debía tributar un gravamen de las características del IG- se constataron como factores de peso para darle fisonomía al proceso conflictivo.

\section{¿En qué aspectos de la problemática tributaria anclaron los actores sindicales sus reclamos?}

En particular, las disputas por el IG se plantearon con respecto a la cuarta categoría, que grava las ganancias obtenidas a través del trabajo personal y comprende a los asalariados registrados de mayores ingresos. Este impuesto es uno de los más relevantes en las estructuras tributarias nacionales para lograr mayor equidad distributiva, ya que su principal característica es la progresividad. Lo que subyace a la idiosincrasia del instrumento es que a mayor ingreso y ganancias de individuos, empresas y sociedades aumenta el monto a ser gravado por el Estado.

El dinero a tributar, en especial para la cuarta categoría, se encuentra directamente relacionado con los resultados de las negociaciones colectivas entre empresarios y trabajadores, con el importe y el ajuste del mínimo no imponible -MNI- por parte del Estado, y con las alícuotas y escalas que estipulan porcentajes diferentes a ser imputados conforme a los ingresos afectados por el impuesto.

El MNI es un elemento de suma centralidad para comprender cómo afecta el gravamen en el ingreso de los trabajadores, ya que determina cuál es el piso salarial que queda exento del pago de ganancias. Este umbral se conforma a través de la suma de conceptos que este puede deducir y, por tal motivo, difiere según su carga familiar y según la posibilidad de justificar otros gastos. Según la lógica de la ley que regula el pago de este impuesto, el excedente está formado por la ganancia extraordinaria del salario, que es aquella que sobrepasa los gastos normales para vivir y mantener un hogar.

Una combinación de variables condujo por tanto a sedimentar el conflicto en torno al pago del IG, fundamentalmente a partir del año 2012. Entre ellas se destacan los aumentos nominales de salarios ocurridos en forma ininterrumpida desde el 2005 en adelante en negociaciones paritarias, ${ }^{11}$ el incremento de la formalización del trabajo, el delos precios de la economía doméstica fruto del proceso inflacionario, ${ }^{12}$ y el atraso en los ajustes del MNI tanto como el congelamiento de las escalas. 
La disconformidad de los trabajadores alcanzados por el IG no es un aspecto novedoso en la historia nacional, como tampoco lo es el gravamen, el cual se legisló en el año 1933 bajo la denominación de impuesto a los réditos, para adquirir finalmente en el año 1973 su denominación actual. Como analiza Sánchez Román (2013), el surgimiento de este impuesto bajo un gobierno dictatorial quedó asociado a un déficit de legitimidad, que en las décadas siguientes por diversas circunstancias resultaría difícil de subsanar. En este marco, los problemas económicos y el régimen de elevada inflación que caracterizó a la Argentina, especialmente entre 1955 y 1976, no asistieron a generar confianza de los contribuyentes con respecto a este instrumento tributario. La inflación, por ejemplo, alteraba la percepción sobre la justeza y la equidad del gravamen, utilizado en aquel tiempo por los tributantes como un argumento para evadir y discutir moralmente la idoneidad del impuesto. En este sentido, y como retomamos a partir del milenio actual, un incremento del ingreso relacionado con la inflación conducía a tributar en una escala más alta cuando no se apreciaba concomitantemente un aumento del salario real.

Si bien las declaraciones o posicionamientos contrarios al pago del impuesto a la renta ya se hicieron presentes en aquellas décadas, no sería hasta la segunda década del 2000 cuando por primera vez sectores del sindicalismo se movilizarían activamente con la consigna de la derogación o modificación del IG, ante un gobierno peronista. Así, las tensiones advinieron en un conflicto sociopolítico peculiar.

Hacia el 2012, cuando los reclamos de los trabajadores por el IG se manifestaron en la calle, se declamaban aspectos distorsivos en el modo de aplicar el impuesto. Entre ellos, se destacaba el hecho de que los trabajadores pasaban rápidamente a una escala más alta con los aumentos paritarios, que respondían en gran medida a la inflación; un piso mínimo de imposición que no acompañaba proporcionalmente el aumento de los precios domésticos (cuestionamientos ya presentes como problema en otras décadas, como consignamos), a lo que se sumaron los efectos de un decreto promulgado en el 2013 por el gobierno (el decreto $n^{\circ} 1.242$ ).

Con aquella normativa quedaron exentos del pago del IG los trabajadores que no habían ganado más que $\$ 15.000$ de sueldo bruto en 2013. Esto último implicó que aquellos que recibieron incrementos salariales desde aquel año pasaban a estar liberados del pago del impuesto, mientras que los nuevos trabajadores que ingresaban al mercado de trabajo con salarios superiores a $\$ 15.000$ bruto tributaban, lo que contribuía en consecuencia a generar un aumento de la desigualdad en el interior de la masa de los asalariados, quienes a igual remuneración bruta podían percibir un ingreso de bolsillo diferente. Las horas extras y los viáticos que percibían los trabajadores también quedaban comprendidos por los descuentos de ganancias, y ello fue percibido muy desfavorablemente en especial por los gremios del transporte.

La evolución del número de los trabajadores alcanzados por el IG desde el inicio de la confrontación abierta en 2012 fue la siguiente:

$\begin{array}{llllll}\text { Año } & 2012 & 2013 & \begin{array}{l}\text { 2014 (luego } \\ \text { del decreto } \\ 1242)\end{array} & 2015 & \begin{array}{l}\text { Febrero } \\ 2016\end{array} \\ & & & & \\ \begin{array}{l}\text { Cantidad de } \\ \text { trabajadores } \\ \text { tributantes del }\end{array} & 1.729 .853 & 2.070 .910 & 1.139 .031 & 1.189 .342 & 1.643 .683 \\ \text { IG n } & & & & \\ \text { promedios } & & & & \\ \text { anuales } & & & & \end{array}$

cuadro elaborado a partir de información citada por chequeado.com ${ }^{13}$ 
El descontento de una parte importante del sindicalismo argentino, que comprendía alrededor de un 10 $\%$ de los asalariados, fue capitalizado por los candidatos a presidente de la oposición, que incorporaron en sus promesas de campaña la eliminación del IG para los trabajadores en relación de dependencia afectados por el gravamen. Así, Sergio Massa, del Frente Renovador, sostuvo durante la campaña presidencial de 2015 que "En la Argentina que viene, los trabajadores no van a pagar más Ganancias" (Diario Clarín, 31/3/ 2015) ${ }^{14}$, proponiendo la eliminación del IG y asegurando que esos ingresos serían compensados con una mayor regulación estatal sobre el juego y la renta financiera. En tanto, Mauricio Macri prometió en la campaña electoral del 2015 eliminar el gravamen para los trabajadores: "El Estado no tiene que quedarse con el fruto de tu trabajo. En mi gobierno, los trabajadores no van a pagar impuesto a las ganancias" (Politica argentina, $2 / 10 / 15)^{15}$.

Por su parte, el candidato oficialista del Frente para la Victoria, Daniel Scioli, incorporó la promesa de modificar el tributo a días de la elección presidencial del 25 de octubre:

Vamos a tomar una medida de fondo en materia de Impuesto a las Ganancias. Un trabajador o jubilado que gane menos de 30 mil pesos netos de bolsillo no va a pagar ese impuesto. Además esta medida se actualizará automáticamente de acuerdo al porcentaje de incremento de movilidad jubilatoria (La Nación, 22/10/2015).

Lejos de perder centralidad temática, con el ascenso de la coalición Cambiemos a la conducción del Estado nacional, el IG continuó ocupando un espacio de relevancia en la agenda sindical y política, si bien la calle dejó de ser uno de los lugares de privilegio para enmarcar la contienda.

\section{CAMBIO INSTITUCIONAL, NUEVOS REPERTORIOS DE ACCIÓN Y CENTRALIDAD PARLAMENTARIA EN LA DISPUTA POR EL IMPUESTO A LAS GANANCIAS}

Durante el último mandato de Cristina Fernández de Kirchner se presentaron una importante cantidad de propuestas por parte de legisladores de la oposición pero también del propio oficialismo que no llegaron a tratarse en el parlamento. La resistencia de la presidenta a modificar el impuesto para atender a las demandas de un sector del sindicalismo se observó como una de las causas que atentaron contra la discusión legislativa de la regulación, según distintas fuentes consultadas.

Preso de su promesa de campaña, Mauricio Macri debió tratar con celeridad el planteo de algunos gremios que reclamaban por la modificación del IG, y ello fue atendido a través de uno de los tantos decretos de necesidad y urgencia que impulsó el gobierno en sus primeros meses de gestión. La modificación parlamentaria de la regulación quedó sin embargo aplazada por el nuevo oficialismo hasta que, en noviembre de 2016, el dirigente opositor Sergio Massa se anticipó al gobierno proponiendo el tratamiento legislativo adeudado a los sindicatos. Ello habilitó un intenso debate y arduas negociaciones para tratar las modificaciones del gravamen.

\section{SINDICALISMO Y MACRISMO EN LA NUEVA ETAPA DE GESTIÓN}

Como destacamos anteriormente, la nueva etapa política implicó una reorganización de las relaciones y alianzas entre actores políticos y sociales. El gobierno nacional mostró en los inicios de su gestión la intención de sostener una relación no conflictiva con los gremios y de control de las protestas sindicales. Hubo gestos en esta dirección, en gran medida como retribución del apoyo en la campaña presidencial que algunos dirigentes - como fue el caso de Hugo Moyano y Luis Barrionuevo, entre otros- habían prestado al candidato de Cambiemos. En esta clave hay que leer la designación de Luis Scervino-integrante del comité de expertos que asesoraba a la CGT liderada por Caló- como superintendente de Servicios de Salud a pocos días de asumir el gobierno, la cual le permitía al sindicalismo controlar los fondos de las obras sociales. El nombramiento fue 
aprobado por Moyano y Barrionuevo, quienes estaban al frente de las otras dos CGT. Asimismo, en el mes de febrero de 2016 otro anuncio retomaba las promesas de campaña: la elevación del MNI, al cual se sumaría en agosto de aquel año la devolución de alrededor de $\$ 30.000$ millones adeudados por el Estado a las obras sociales que gestionan los sindicatos.

Por tanto, el gobierno intentó establecer un vínculo de armonía con los gremios con la intención de controlar la calle, en un período en el cual las protestas y las manifestaciones colectivas se producían de modo frecuente. Dichas acciones fueron encaradas por movimientos sociales, gremiales y de la sociedad civil contra medidas políticas que se orientaban a un ajuste de las cuentas públicas, y contra el llamado "tarifazo", que implicó un aumento sustantivo de las tarifas de servicios esenciales, principalmente la del gas. ${ }^{16}$

Estos intentos conciliadores por parte del gobierno con la dirigencia sindical convivieron con un significativo descontento de los sectores del trabajo por las políticas de empleo y de ingresos encaradas por la presidencia. En este marco, la unificación de la CGT se auguraba con mayor contundencia a instancias de estas medidas de gobierno, de cara a los congresos confederales que ungirían una nueva conducción de consenso el 22 de agosto. También las dos CTA, enfrentadas por su relación con el kirchnerismo, articularon sus esfuerzos reivindicativos en el nuevo contexto político.

¿Qué ocurrió entonces con el conflicto sociopolítico en torno del impuesto a las ganancias? Como destacamos anteriormente, en el marco de un conjunto de decretos de necesidad y urgencia que el gobierno de Macri instrumentó en los primeros cien días de su gobierno se anunció el 18 de febrero de 2016 la suba del MNI a 30.000 pesos en bruto (Decreto 394/2016). Así, los asalariados con hijos comenzaban a pagar el impuesto a partir de 25.000 pesos de bolsillo, en tanto que los solteros lo harían a partir de 18.880 pesos netos. La medida no alcanzó sin embargo a las escalas y las alícuotas que afectaban al tributo, modificaciones que, según el presidente, serían pospuestas para el 2017. ${ }^{17}$ Tampoco se anunciaron modificaciones para los monotributistas. ${ }^{18}$

Pero el otro cambio importante del decreto presidencial se vinculaba con la derogación de la norma (1247) que en 2013 había instaurado un parte aguas entre los trabajadores alcanzados por ganancias. Aquella normativa implementada por el gobierno de Cristina F. de Kirchner había profundizado un aspecto inequitativo del impuesto, al generar un mecanismo desigual de recaudación al interior de la masa asalariada. No obstante, al derogarse esta norma se producía una alteración que acentuaba la complejidad del proceso: por un lado, el cambio del MNI alivianaba el peso del gravamen fundamentalmente en las escalas más bajas de tributación. Sin embargo, y al mismo tiempo, generaba un incremento de la masa de trabajadores que quedaba incluida en el pago del tributo. Así, según distintas fuentes, ascendió la masa de tributantes a 1.800.000 trabajadores aproximadamente.

El oficialismo y los partidos de la oposición reconocían sin embargo la necesidad de transformar legislativamente las escalas y alícuotas, debido el trazo insuficiente en la modificación del Poder Ejecutivo sobre el MNI, tal como demandaban los actores sindicales. En los primeros días de marzo hubo encuentros entre los dirigentes de las tres CGT (Caló, Moyano y Barrionuevo) en una foto que anticipaba la unificación de la organización sindical. El reclamo por la modificación de la ley del IG ocupaba un lugar destacado en sus agendas de unidad, y permitió avanzar en una alianza táctica entre legisladores de extracción sindical de distintos bloques para forzar la presentación de un proyecto de ley que modificara el MNI y las escalas del tributo.

El 29 de abril tuvo lugar la primera manifestación conjunta de agrupamientos sindicales durante la era Cambiemos. Esta acción colectiva fue impulsada por las tres CGT y las dos CTA, y estuvo acompañada por organizaciones de izquierda, movimientos sociales, cooperativas de la economía social, gremios independientes y el Partido Justicialista, entre otros. La principal demanda: la crisis en el empleo y los despidos. Dos días antes de la movilización, una ley antidespidos había recibido la media sanción en el Senado. Sin embargo, con posterioridad a la marcha del 29, el presidente vetó esa ley. A pesar del descontento 
gremial, no se produjo otra medida de fuerza tal como habían exigido algunas organizaciones participantes de la marcha.

En este marco, la estrategia sindical mutó de la calle al palacio. La reiterada posición confrontativa, que ganó visibilidad con paros y movilizaciones durante el gobierno kirchnerista, fue reemplazada por una modalidad de presión que alternó la presentación de iniciativas legislativas a través de diputados de extracción gremial con una permanente negociación con el Ejecutivo Nacional, en la que este buscó tener como interlocutor privilegiado a la CGT, sin convocar a ninguna de las dos CTA.

\section{SOBRE COALICIONES PARLAMENTARIAS}

Si bien en los sistemas presidenciales el primer mandatario no depende de la existencia de una mayoría parlamentaria para gestionar, es necesario que disponga de apoyos legislativos suficientes para aprobar sus iniciativas. En la formación de una coalición legislativa influyen el control efectivo que el Ejecutivo tiene sobre el Legislativo, los grados de fragmentación y polarización de las cámaras y la cohesión y disciplinas partidarias, como también de qué nivel de representación tienen los partidos con los que se busca coordinar legislativamente en el gabinete (Clérici, 2017). Las reglas y los incentivos institucionales, como el sistema electoral y la posibilidad de reelección de los legisladores, son variables que influyen en el comportamiento de estos (Carey, 2007; Clérici, 2018), así como también su ideología (Morgenstern, 2004).

Las legislaturas de América Latina son en general multipartidarias, sin mayorías consolidadas que superen el $50 \%$ de las bancas. En estos escenarios de fragmentación, los partidos gobernantes deben estructurar coaliciones con partidos minoritarios para conseguir la aprobación de sus iniciativas legislativas (Calvo, 2014). Esta búsqueda de ampliar los acuerdos legislativos estuvo presente en todos los presidentes argentinos desde el retorno de la democracia. Ni Raúl Alfonsín ni Carlos Menem dispusieron de mayorías absolutas en el Congreso. Sin embargo, esta condición no obstaculizó un importante nivel de aprobación de las iniciativas legislativas promovidas por el Poder Ejecutivo. Si bien en la gestión del ex presidente Menem prevaleció la imposición de decretos por sobre la participación del Congreso para llevar adelante las reformas estructurales de corte neoliberal, su gobierno debió construir coaliciones legislativas que legitimaran esas políticas, por ejemplo, en el caso de las privatizaciones (Etchemendy y Palermo, 1998). En esta etapa de gobierno los niveles más altos de productividad legislativa se alcanzaron entre los años 1993 y 1997, cuando el menemismo logró consolidar una coalición legislativa que incluía a un Partido Justicialista ya alineado en la coalición de gobierno (Calvo, 2007).

A la inversa, la falta de mayoría legislativa propia de un presidente puede ser leída como una dificultad para avanzar con el proceso decisorio del Ejecutivo. Este fue el caso del presidente Néstor Kirchner cuando asumió en mayo del 2003, dado que el peronismo estaba fracturado en tres bloques: PJ duhaldista-kirchnerista, Frente Justicialista Azul y Blanco-menemista y Frente Movimiento Popular (RodriguezSaá). Como señalan Alejandro Bonvecchi y Javier Zelaznik, (2006) la construcción de una nueva mayoría oficialista como base de sustentación política del proyecto kirchnerista avanzó muy rápidamente, al incorporarse al bloque diputados menemistas, y otros no peronistas pero afines al presidente. Si bien después de las elecciones de octubre del 2005 -tras la fractura con el duhaldismo-el ex presidente contó con un contingente legislativo por debajo del umbral mayoritario, nuevamente logró recomponer esa mayoría incorporando diputados provenientes de bloques minoritarios y, ocasionalmente, gracias al apoyo del Peronismo Federal y bloques provinciales (Bonvecchi y Zelaznik, 2006).

La Alianza Cambiemos no contó con mayorías parlamentarias al inicio de su gestión presidencial: de las 257 bancas que componen la Cámara de Diputados pertenecían al oficialismo 92 escaños ( 44 del PRO, 42 de la Unión Cívica Radical y 6 de la Coalición Cívica). Sin mayoría visible en la cámara baja, las dos fuerzas predominantes -Cambiemos y el Frente para la Victoria- tenían composición coalicional (Zelaznik, 2015), lo cual obligaba a estos espacios políticos a aceitar mecanismos de resolución de conflictos internos. 
En este marco, a poco de iniciarse el 2016, el gobierno logró su primer éxito parlamentario al aprobarse la derogación de las leyes "cerrojo" y "de pago soberano" ${ }^{19}$, que habilitaban el pago a los "fondos buitres". El proyecto fue aprobado en Diputados por 165 votos a favor contra 86 votos negativos. Además de los legisladores de la coalición, una vez incorporadas las modificaciones al texto original el proyecto contó con el apoyo de los 34 legisladores del Frente Renovador (FR) ${ }^{20}, 27$ del Bloque Justicialista (BJ) y aliados, el Frente Amplio Progresista (Partido Socialista y GEN, entre otros) y partidos provinciales como el Movimiento Popular Neuquino (MPN) y santiagueños del Frente Cívico. En tanto, el proyecto de ley fue rechazado por 77 votos del Frente para la Victoria (FPV), 4 de Libres del Sur, 1 de Proyecto Sur y 4 del Frente de Izquierda y los Trabajadores. La iniciativa del Poder Ejecutivo fue ratificada sin modificaciones a fines de marzo en el Senado con 54 votos a favor y 16 en contra. Allí los legisladores de Cambiemos obtuvieron el respaldo del bloque FPV liderado por Miguel Pichetto, el Frente Renovador, el Peronismo Federal, el GEN, y de partidos provinciales.

El 14 de junio de 2016, Diputados dio media sanción a la denominada "ley ómnibus", un proyecto que contenía diferentes iniciativas del Poder Ejecutivo como un blanqueo de capitales, un régimen de regularización de deudas tributarias, la eliminación de gravámenes a los activos de personas de mayor patrimonio (bienes personales), la privatización encubierta de participaciones accionarias en poder de Anses, y la legitimación de los acuerdos firmados con los gobernadores para la restitución paulatina del 15 por ciento de la coparticipación que se le asignaba a Anses. El oficialismo obtuvo un holgado resultado de 162 votos a favor (Cambiemos, FR, Bloque Justicialista y algunos legisladores provinciales) y 76 en contra (FPV, la izquierda del FIT, y el solitario voto de Alcira Argumedo, de Proyecto Sur). En el Senado, el oficialismo se impuso con 55 votos a favor (Cambiemos, parte del FpV conducido por Miguel Pichetto y algunos bloques provinciales y el PJ no kirchnerista) y 11 en contra (kirchnerismo y Lucila Crexell del MPN), con la única abstención de "Pino" Solanas ${ }^{21}$.

\section{LA MODIFICACIÓN LEGISLATIVA DEL IMPUESTO A LAS GANANCIAS}

Si bien en los últimos años la representación gremial en el parlamento no superó la docena de legisladores, varios de los proyectos legislativos que se presentaron en la Cámara de Diputados durante el gobierno kirchnerista fueron promovidos por legisladores de extracción sindical como Facundo Moyano y Omar Plaini (FPV), o cercanos al sindicalismo, como Héctor Recalde, quien a su vez presidió el bloque del FPV en la primera parte del gobierno de Cambiemos $(2015-2017)^{22}$. En tanto, Oscar Romero, dirigente del Sindicato de Mecánicos y Afines del Transporte Automotor (SMATA), presidió el bloque justicialista y acompañó las propuestas de cambios en el tributo, mientras que el triunviro y dirigente del Frente Renovador Héctor Daer tuvo una participación muy activa en las negociaciones con el gobierno nacional en torno a las modificaciones del gravamen. A pesar del interés de estos y otros legisladores por impulsar cambios, la formación por parte de Cambiemos de una coalición ampliada a un sector de la oposición durante el 2016 operó exitosamente en el caso de la modificación del impuesto a las ganancias.

En noviembre de 2016 Sergio Massa presentó un proyecto de su bloque para impulsar cambios en el IG. Así, el líder del Frente Renovador construyó una nueva oportunidad política para reposicionarse en la agenda pública y obligar al gobierno a adelantar el tratamiento del proyecto. Como respuesta a la jugada de Massa, el gobierno ese mismo mes envió su propio proyecto sobre IG con modificaciones en escalas y alícuotas, y un moderado aumento del MNI del orden del $15 \%$, porcentaje inferior a la inflación proyectada para el 2017. De este modo, el MNI para los trabajadores casados con dos hijos se fijaba en 25.231 pesos netos mensuales (era en ese momento de 25.000 pesos el MNI), y en 21.712 para trabajadores solteros. Se modificaban además las alícuotas del gravamen con una escala mínima del $5 \%$, mientras que el tope de la escala se mantenía en $35 \%{ }^{23} \mathrm{El}$ proyecto, enviado sin ninguna negociación previa con la oposición más dialoguista, generó un 
fuerte rechazo de los diferentes bloques legislativos, lo que dio el marco para que Massa recuperara centralidad opositora al proponer modificaciones de mayor alcance que las propuestas por el oficialismo. ${ }^{24}$

Tras una polémica entre el gobierno y el exintendente de Tigre, en la que este último fuera acusado de ser el "político menos confiable" por parte del jefe de gabinete Marcos Peña, el Frente Renovador y el Frente para la Victoria, junto con el bloque justicialista y el apoyo de otros bloques minoritarios, consensuaron un proyecto que elevaba el MNI a 44.000 pesos para los casados con dos hijos y a 35.000 para los solteros. ${ }^{25}$ La actualización del MNI mantenía un criterio propuesto por los diputados kirchneristas y aceptado por el resto de los bloques opositores: solo pagaría el $10 \%$ de los asalariados que más ganaban. Asimismo, se excluía del impuesto el medio aguinaldo, las horas extras, bonos por productividad y a los jubilados que ganaran hasta 60 mil pesos mensuales; se creó adicionalmente una deducción por zona desfavorable para provincias patagónicas. El proyecto contemplaba compensar el costo fiscal de la reforma impositiva con un impuesto del 7,5\% para el juego online y del $10 \%$ para máquinas tragamonedas, gravar los plazos fijos por encima de 1,5 millones de pesos y la operatoria con títulos públicos -las Lebac-, así como imponer la remisión de dividendos al exterior. A esto se agregaba dar marcha atrás con la quita de retenciones a las empresas mineras.

Pablo Kosiner, diputado integrante del bloque Justicialista, consideró que la promesa de eliminar el impuesto por parte del gobierno en la campaña, y el posterior desinterés por consensuar un proyecto, fueron los factores que desencadenaron la presentación de un proyecto alternativo:

¿Por qué se termina de precipitar todo? El mismo Gobierno colocó como eje de su campaña “que nadie va a pagar ganancias”. Y en política si vos pusiste la pelota en juego, tenés que sostenerla... Pero, por otro lado, las provincias decían bueno, "cumplilo, pero fijate cómo lo cumplís, porque nos estás complicando a nosotros” (....) ¿Por qué termina saliendo este proyecto alternativo? Porque el Gobierno presentó un proyecto y dijo: "este es el mayor esfuerzo que puede hacer el Gobierno", y no lo abrió a modificaciones. Si hay alguien responsable de haber juntado a toda la oposición, es el Gobierno (P. Kosiner, comunicación personal, mayo de 2017).

La sesión del 6 de diciembre del 2016 fue objeto de un intenso debate en torno al proyecto consensuado por los principales bloques legislativos opositores. El oficialismo organizó su exposición planteando la prioridad que tenía la modificación de las escalas sobre el MNI a la hora de volver más progresivo el tributo: "ese es el corazón de la reforma que estamos proponiendo", expresó Luciano Laspina, presidente de la Comisión de Presupuesto de la Cámara de Diputados. El legislador santafecino marcó la importancia de mantener un equilibrio entre la progresividad de las escalas y la racionalidad en el costo fiscal. Sostuvo que las modificaciones incorporadas eran solicitadas por todo el arco opositor, así como por diferentes sectores sociales, y que representaban un costo para el erario público de 55 mil millones de dólares. Finalmente, presentó la reforma del oficialismo como reparadora de la "injusticia" creada por el gobierno anterior, no solamente con el congelamiento de las escalas sino también con los cambios del año 2013, los cuales definía como una "aberración jurídica y tributaria que violaba el artículo 14 bis de la Constitución Nacional” (Honorable Cámara de Diputados, versión taquigráfica del 6 de diciembre de 2016, pp. 129-133). Acusó así a la oposición de plantear una discusión política, no técnica, y de irresponsabilidad por proponer una reforma que desfinanciaba a las provincias (Honorable Cámara de Diputados, versión taquigráfica del 6 de diciembre de 2016, pp. 134-149).

Mientras el eje de Cambiemos estuvo puesto en la progresividad de las escalas, el Frente para la Victoria, a través de la intervención de Axel Kicillof, colocó el foco en el mínimo fijado por el gobierno durante marzo del 2016 en 22.000 pesos, que provocó que:

de un millón de trabajadores que pagaban impuesto a las ganancias cuando nosotros terminamos el gobierno, se pasara a que hoy lo paguen prácticamente el doble. Esto no es solo no cumplir una promesa, es no cumplirla doblemente, porque lo que ha hecho es duplicar la cantidad de argentinos que pagan el impuesto a las ganancias con respecto al año 2015, que tanto se ocupan de denostar. 
El ex ministro recordó el criterio mantenido durante el gobierno anterior de que solo pagaran el impuesto el 10 por ciento de los trabajadores registrados. Además, Kicillof definió como "estafa electoral” la promesa hecha durante la campaña del presidente Macri de que los trabajadores no iban a pagar impuesto a las ganancias durante su gobierno. ${ }^{26}$ Con críticas a la quita de impuestos a los sectores más poderosos de la sociedad y a la devaluación del 2016, Kicillof respondió, frente a la calificación de "irresponsable" del proyecto articulado entre el FVP y el Frente Renovador, que

la verdadera responsabilidad es cumplir con las propias ideas, convicciones y la plataforma electoral. Por eso durante la campaña electoral nuestro candidato no dijo que iba a eliminar el impuesto a las ganancias, sino que iba a modificar las escalas y el mínimo no imponible.

Luego de haber reconocido la flexibilidad de posturas para arribar a un acuerdo opositor, y de asegurar que el proyecto no sería lesivo para las finanzas provinciales, el legislador kirchnerista justificó el proyecto de ambos bloques opositores como un estímulo al consumo y a la reactivación productiva: "con la frente en alto podemos defender los derechos de los trabajadores, de las provincias y de todo el pueblo argentino".

En línea con lo planteado por Kicillof, el legislador del Frente Renovador Marcos Lavagna consideró: "el proyecto del ley enviado por el Ejecutivo incorpora nuevos trabajadores y que esto es inadmisible, como también lo es que no se grave la renta financiera". Con un tono más conciliador que el del exministro de economía, Lavagna reafirmó la "vocación para encontrar consensos que ha caracterizado al Frente Renovador durante el año parlamentario" y lamentó la falta de diálogo del gobierno, ${ }^{27}$ reconociendo algunos aspectos positivos del proyecto oficialista como las modificaciones en las escalas. Lavagna coincidió con Kicillof en que su proyecto motorizaba el mercado interno, las PyMES, e incentivaba a los sectores ligados a la producción, lo cual que permitiría generar nuevo empleo. Sostuvo que el costo fiscal del proyecto que proponían era solo 10 mil millones de pesos más que el del gobierno -60.000 contra 50.000 millones de pesos-, que fue consensuado con los gobernadores y que sería compensado con las imposiciones a las rentas financieras que no tuvieran destino productivo. "Si dios quiere saldremos de este recinto con una norma que proteja a los trabajadores, a los jubilados, a los monotributistas, a los autónomos y dé tranquilidad a las provincias de que no van a perder un peso de sus recursos", concluyó (Honorable Cámara de Diputados, versión taquigráfica del 6 de diciembre de 2016, pp. 139-141).

Luciano Laspina reconoció que en el debate se impuso la centralidad del MNI por sobre la modificación de las escalas:

En definitiva el debate se perdió. En términos de que para amplios sectores del arco político, de la CGT, el mínimo no imponible era la cuestión fundamental y no las escalas, que es lo que realmente le da más progresividad a todo el impuesto. Con el primer reacomodamiento del mínimo no imponible, nosotros pensamos que ya estábamos bien en ese tema, que por lo menos habíamos eliminado una parte importante de la distorsión y que de aquí en adelante había que trabajar sobre las escalas. Para que haya una progresividad entre los que pagan impuestos, sabiendo que el impuesto alcanzaba al $10 \%$ de los trabajadores con más altos ingresos (L. Laspina, comunicación personal, febrero de 2017).

Después de un acalorado debate, la iniciativa opositora recibió media sanción en Diputados, con 140 votos positivos, 86 negativos y 7 abstenciones. El proyecto fue remitido entonces al Senado.

\section{NuEvo ESCENARIO DE NEgOCIACIÓN: gobERNADORES, CGT Y "PERONISMO RACIONAL"}

En la Cámara Alta el gobierno encontró un ámbito propicio para avanzar con las conversaciones tendientes a construir un consenso en torno a la necesidad de incorporar modificaciones al proyecto de Diputados. En ese marco, mantuvo como interlocutor privilegiado al jefe del bloque del FPV, Miguel Angel Pichetto. El primer traspié para la oposición fue el dictamen en la comisión de Presupuesto del Senado: el mismo alcanzó solo cinco firmas, cuatro menos que las necesarias por convertirse en despacho de mayoría y así avalar el proyecto enviado desde Diputados. ${ }^{28}$ La votación reflejó fricciones entre los senadores kirchneristas que acompañaban 
el proyecto opositor y los senadores peronistas sensibles al interés de varias provincias de que no se vieran afectados los recursos coparticipables que recibían de la nación. ${ }^{29}$

En el horizonte de posibilidades del gobierno se barajaron las alternativas de consensuar un proyecto con un costo fiscal más bajo o bien enfrentar a la oposición con una propuesta acordada previamente con los gobernadores. Con algún grado de flexibilidad sobre la posibilidad de implementar un impuesto al juego, el oficialismo se mantuvo reticente a la reposición de retenciones a la minería y la soja, así como a gravar la renta financiera. A medida que se debatía en el Senado, en una mesa chica el gobierno acordó con la conducción de la CGT incorporar los reclamos sobre los que el triunvirato ${ }^{30}$ tenía principal interés, especialmente los de los gremios que integraban la Confederación Argentina de Trabajadores del Transporte (CAAT): las deducciones por horas extras, viáticos y feriados.

Así, avanzando en negociaciones con los actores extraparlamentarios, el gobierno logró, a través de la presión sobre un eventual recorte de los recursos coparticipables a las provincias, que un numeroso grupo de gobernadores se pronunciara en contra del proyecto opositor girado al Senado. Los más enfáticos fueron los representantes del espacio Cambiemos: Horacio Rodríguez Larreta (CABA), Alfredo Cornejo (Mendoza), Ricardo Colombi (Corrientes) y Gerardo Morales (Jujuy). También lograron alinear a gobernadores del PJ como Juan Manuel Urtubey (Salta), Gustavo Bordet (Entre Ríos), Roxana Bertone (Tierra del Fuego) y Juan Schiaretti (Córdoba). A ellos se sumaron Omar Gutiérrez del Movimiento Popular Neuquino, Alberto Weretilneck de Juntos Río Negro y Hugo Passalacqua (Misiones). En esta misma línea de oposición al proyecto de Diputados se pronunciaron los mandatarios de las provincias "mineras" como fue el caso de San Juan. Su gobernador, Sergio Uñac, consideró negativa la iniciativa porque volvía a gravar la renta minera para no desfinanciar al Estado: "la restitución de las retenciones a la minería afectaría a un sector que integra más de 40 mil familias, entre trabajadores directos e indirectos en San Juan” (Página 12, 10/12/2016). Mientras el gobernador de Santa Fe, Miguel Liftschitz, fue más moderado en la crítica, otros jefes provinciales como el pampeano Carlos Verna apoyaron el proyecto con medio dictamen convalidado en Diputados. No obstante, solo el gobernador de Chubut, Mario Das Neves, estuvo presente en la sesión del Senado. Aliado de Sergio Massa en ese momento, Das Neves había denunciado los días previos: "comienza a operar la disciplina de la chequera, empiezan los llamados telefónicos, empieza a retacearse la transferencia de fondos” (Página 12, 10/12/2016).

En cuanto a los aspectos técnicos del proyecto que reelaboró el Senado, las modificaciones introducidas en la comisión de Presupuesto en relación a la elevación del MNI, el cambio y la actualización de las escalas, así como las diferentes deducciones incorporadas fueron valoradas y resaltadas por distintos legisladores de las bancadas oficialista y opositora en su mayoría. ${ }^{31}$ Una medida reconocida especialmente por los senadores de las provincias patagónicas fue el aumento de las deducciones en un $22 \%$ para los trabajadores de esa región por considerarla "zona desfavorable" ${ }^{32}$. En cambio, la eliminación de las retenciones a la minería, a la soja y a la renta financiera que impulsaba el proyecto opositor de Diputados fue objeto de críticas entre diferentes senadores del peronismo -kirchnerista y no kirchnerista-, pero también de otros legisladores como Fernando Solanas (Proyecto Sur) y de María Elena Odarda (UNEN, ligada a la Elisa Carrió).

Los posicionamientos críticos de los senadores tuvieron dos destinatarios previsibles: el gobierno kirchnerista y la gestión en curso. En el primer caso se cuestionaban los aumentos por decretos del MNI y el congelamiento de las escalas (Guillermo Pereyra, Daniel Lovera) en sus aspectos técnicos, y la falta de diálogo y de voluntad para abordar el tema a pesar de las propuestas de la oposición (Luis Luenzo). El propio Abal Medina reconoció, en una elíptica crítica a un sector político del que ya no formaba parte, que "todos estamos en mora, tanto el oficialismo que prometió en campaña eliminar el IG y hoy no lo estamos eliminando y eso es un problema del oficialismo", como también el gobierno anterior, dado que "es cierto que durante doce años de gobierno no tocamos las escalas y no levantamos el MNI" (Senado de la Nación, versión taquigráfica del 21 de diciembre de 2016, p. 7). Más aún, se reconocieron los costos políticos de esa decisión: "nos equivocamos en no elevar el MNI y perdimos la elección (Daniel Pérsico). 
Pero también el debate sobre ganancias fue el marco que habilitó un cuestionamiento más general sobre la política impositiva vigente, considerada en muchos casos como injusta y regresiva, incluso para algunos legisladores afines al gobierno o moderadamente opositores. ${ }^{33}$ Desde el punto de vista político, el kirchnerismo cuestionó, a través del senador Marcelo Fuentes, el viraje que dio el Frente Renovador para apoyar el actual proyecto con modificaciones:

Y en esta división que hubo en dos semanas, súbita, en políticos estadistas, constructivos del equilibrio fiscal y chantas, mentirosos y demagogos que posteriormente en un solo día cambian e intercambian menú conjunto, resulta que el hombre menos confiable de la política luego se convierte, dos días después, en el garante de lo que hoy se va a tratar acá (Senado de la Nación, versión taquigráfica del 21 de diciembre de 2016, p.41).

Luego de una semana de febriles negociaciones, el Senado aprobó el 21 de diciembre el proyecto con modificaciones a la ley de impuesto a las ganancias. Con 56 votos a favor, 12 abstenciones y 2 votos en contra, la Cámara Alta incorporó cambios a la media sanción proveniente de Diputados. ${ }^{34}$ De este modo, el proyecto se reenvió a Diputados con sus respectivas modificaciones: se elevó el MNI a 37.000 pesos para casados con dos hijos y a 27.941 pesos para trabajadores solteros, se mantuvieron las deducciones por hijo hasta 24 años y se eximieron de ganancias a los jubilados que cobraran un salario equivalente a 6 jubilaciones mínimas. ${ }^{35}$ Asimismo, quedó exento el aguinaldo para aquellos salarios que no superaran los 37.000 pesos brutos en caso de trabajadores casados con dos hijos. También se aplicó una deducción a los alquileres hasta un $40 \%$ con tope de 4.000 pesos mensuales para aquellos contribuyentes que no fueran propietarios en forma total o parcial de un inmueble. A su vez, se incorporaron deducciones de un $40 \%$ en viáticos para determinadas actividades (con un límite anual de 20.787 pesos) y para horas extras. ${ }^{36} \mathrm{~A}$ esto se sumó el incremento en un $22 \%$ del MNI y las deducciones para las provincias patagónicas, incluida La Pampa. ${ }^{37}$

Por otra parte, se pasó de un esquema de 7 a 9 escalas; se incorporó una nueva escala con una alícuota menor a la primera de la tabla anterior. ${ }^{38}$ Las modificaciones implementadas en el proyecto de ley suprimieron la creación de un impuesto a la minería y a la renta financiera propuestas en la media sanción de Diputados. En cambio, se gravaron con el 0,75\% las apuestas en tragamonedas y con el 2,5\% las apuestas online. Asimismo, se estableció un incremento en la tributación de la explotación de juegos de azar que pasaba del 35 al 41,5 $\%$. Se determinó aplicar, por única vez, un impuesto del $15 \%$ a las rentas de operaciones especulativas con dólar futuro (Ámbito Financiero, 22/12/2016).

Laspina reconoció la posición negociadora y determinante del Senado para modificar el proyecto con media sanción de Diputados:

El Senado prende todas las luces de alerta para los gobernadores. Lo que iba a pasar es que el Senado se iba a hacer responsable del proyecto que había salido acá, que era infinanciable. O lo frenaba y lo modificaba totalmente, o si lo aprobaba como estaba, iba a tener que ser vetado, porque nos fundíamos si se aprobaba ese proyecto. Prácticamente hacía desaparecer el impuesto a la ganancia de la cuarta categoría. A partir de ahí se abre una mesa de negociación, que incorporó a los principales líderes del Senado, a los principales líderes de la CGT y a los gobernadores. Y entre esas tres patas o cuatro, CGT, Senado, Gobierno y gobernadores, se consensuó llegar a una cosa bastante más racional. En esa negociación, el mínimo no imponible subió más de lo que nosotros hubiésemos pretendido y las escalas se hicieron un poco menos progresivas de lo que nosotros hubiésemos pretendido. 39

Finalmente, el proyecto acordado entre el gobierno y la CGT fue aprobado el 22 de diciembre en la Cámara de Diputados. Obtuvo 166 votos a favor, 5 en contra y 3 abstenciones. Apoyaron el proyecto de Cambiemos el Frente Renovador, el Bloque Justicialista, el GEN de Stolbizer, el Movimiento Evita y Alicia Ciciliani por el socialismo santafesino. Se opuso el Frente de Izquierda y se abstuvieron los diputados de Compromiso Federal (Rodriguez Saá) y de Proyecto Sur. En tanto, el FPV se retiró del recinto con excepción de cuatro diputados sanjuaninos que votaron a favor, entre los que se encontraba el vicepresidente del Partido Justicialista, José Luis Gioja (Página 12, 23/12/2016) ${ }^{40}$.

Omar Maturano, secretario general del sindicato de ferroviarios La Fraternidad, uno de los gremios de transporte que integra la CATT, ofrecía una mirada más escéptica sobre lo que originalmente se había pactado y sobre el resultado final de la negociación; también puso en duda los intereses perseguidos por algunos actores intervinientes en el acuerdo: 
Se había arreglado todo con los diputados y los senadores de todos los bloques. Fueron horas extras y viáticos. Nos faltaban los días feriados. Y estábamos contentos, era mucha plata. Nosotros, por ejemplo, las horas extras las cobramos al 50 por ciento más. Cuando vamos a discutir, nos dicen "que sí, que está todo bien”. El tema es que nosotros no estuvimos en el despacho final de comisión. Los llamaron a ellos, a la CGT, y arreglaron todo; desarreglaron todo mejor dicho. Entró Massa, entraron otros. En el zafarrancho fueron los "gordos" y cerraron que no nos den a nosotros lo que habíamos solicitado. Nos hicieron una encerrona... Después empezaron a tallar los gobernadores; [el impuesto] es coparticipable y los gobernadores les dieron instrucciones también a los diputados de que no accedan porque les quedaba menos plata. Y eso también diluyó el primer acuerdo. Los gobernadores son también los culpables ¿no? (O. Maturano, comunicación personal, julio de 2017).

\section{A MODO DE CIERRE}

La problemática en torno al impuesto a las ganancias se convirtió en un conflicto que atravesó la agenda política durante casi todo el segundo mandato de Cristina Fernández de Kirchner. Si bien las discoformidades con el pago de un impuesto de las características del IG ya se habían notado en otros momentos históricos, en el actual milenio las tensiones confluyeron en la dinamización de un conflicto sociopolítico de características singulares. En este enfrentamiento, una porción de los gremios y el gobierno que había gestado políticas públicas dirigidas a mejorar las condiciones de la clase trabajadora fueron los principales actores de la disputa. Pero a ellos se sumaron partidos políticos de la oposición que intentaron obtener réditos electorales de la contienda de cara a la transición presidencial de 2015. La dificultad de la presidenta y del parlamento para dar lugar a una revisión del impuesto que atendiera alguno de los reclamos del sindicalismo condicionó por tanto al siguiente gobierno a lidiar con aquella problemática. Si se tienen en cuenta las promesas de campaña, cualquiera resultara el dirigente triunfante tendría que tratar con las demandas por el IG.

Con el objetivo de encontrar una solución para encauzar la contienda, y tras haber realizado una intervención puntual sobre los valores del MNI y el decreto 1242 en los primeros cien días de su gestión, el gobierno de Cambiemos inició en consecuencia una serie de negociaciones con actores parlamentarios y extraparlamentarios que culminó en un triunfo legislativo importante, desde una posición de debilidad inicial en el Parlamento. Así logró imponer un proyecto que elevó el MNI e incorporó, luego del debate y de la presencia y presión de otros proyectos opositores, nuevas deducciones que favorecerían a los sectores asalariados alcanzados por el gravamen.

En este marco, se implementó una actualización anual que, si bien representó una mejora al congelamiento anterior, significó un porcentaje inferior conforme a la relación entre el índice de inflación y el promedio resultante del RIPTE (Remuneración Imponible Promedio de los Trabajadores Estables). Las modificaciones de las escalas no fueron sustanciales y se mantuvo congelada en $35 \%$ la alícuota que grava la escala más alta, es decir, a los sectores de mayores ingresos.

Asimismo, no se diferenció el impuesto para las personas jurídicas, aspecto observado críticamente por algunos tributaristas. Allí, la alícuota es única y plana (para todo nivel de ganancia) del 35 \%: pagan lo mismo todas las empresas, sin distinción de tamaño o según reinviertan o no sus utilidades. Una reforma integral debería haber modificado este aspecto, con alícuotas diferenciales según tamaño, utilidades normales o extraordinarias y alícuotas más altas a quienes distribuyeran las ganancias en dividendos (Foro Universitario de Estudios Fiscales, 2016) ${ }^{41}$.

En términos políticos, el proyecto de ley aprobado en el parlamento reflejó la exitosa construcción coalicional por parte del oficialismo, al incorporar en los acuerdos a un sector de la oposición referenciado en legisladores alineados con diferentes gobernadores. Esta estrategia ya le había permitido al gobierno obtener importantes resultados en el tratamiento de otras leyes significativas a lo largo del 2016 como las ya mencionadas derogaciones de la ley cerrojo y de pago soberano, y la ley ómnibus. En segundo orden, el gobierno también demostró eficacia en la secreta negociación con el triunvirato de la CGT -en el que dos integrantes estaban alineados con el Frente Renovador-, aun cuando el resultado de la misma dejó pendientes algunos reclamos de particular interés para los gremialistas del transporte. 
En relación a la oposición, el tratamiento de ganancias reposicionó a Sergio Massa en el centro de la agenda pública y expresó un primer intento, malogrado luego cuando el Frente Renovador terminó aprobando el proyecto con modificaciones del Senado, de articulación opositora en la Cámara de Diputados. El gravamen favoreció así un primer acercamiento de las fuerzas opositoras en la Cámara de Diputados, que, con sus diferentes vaivenes, se consolidaría en diciembre del 2017 con el rechazo conjunto al proyecto oficialista de reforma previsional, aprobado por una mínima diferencia de votos en un marco de fuerte movilización social y represión policial.

\section{REFERENCIAS}

Armelino, M. (2012). Kind of blue. Las vicisitudes de la Central de Trabajadores de la Argentina (CTA) durante los años kirchneristas. En G. Pérez y A. Natalucci (eds.), Vamos las bandas. Organizaciones y militancia kirchnerista (pp. 101-126) Buenos Aires: Nueva Trilce.

Bonvecchi, A., y Zelaznik, J. (2006). La construcción de la mayoría legislativa kirchnerista. Buenos Aires: Fundación PENT.

Brito, G., y Calderón, J.(2016). Balance político de un año PRO. Celag, 15 de diciembre. Recuperado en: http://www .celag.org/informe-de-analisis-politico-un-ano-pro/

García Ramos, J. M. (1992). Recursos metodológicos en la evaluación de programas. Bordón, 43, 461-476.

Calvo, E. (2007). The Responsive Legislature: Public Opinion and Law Making in a Highly Disciplined Legislature. British Journal of Political Science, 37(2), 263-280.

Calvo, E. (2014). Legislator Success in Fragmented Congresses in Argentina: Plurality Cartels, Minority Presidents, and Lawmaking. Nueva York: Cambridge University Press.

Carey, J. (2007). Competing Principals, Political Institutions, and Party Unity in Legislative Voting. American Journal of Political Science, 51(1), 92-107.

Clérici, P. (2017). Coaliciones al cuadrado. Las coaliciones políticas como fenómeno a explicar y como variable independiente. Foro de Debate. Revista Iberoamericana XVI(64), ISSN: 0034-9631, 237-243.

Clérici, P. (2018). La influencia de la dicotomía oficialismo/oposición sobre la congruencia de las coaliciones electorales en Argentina. Postdata 23, 1, ISSN 1515-209, 121-149.

Delfini, M., y Ventrici, P. (2016). Qué hay de nuevo en el sindicalismo argentino? Relaciones laborales y reconfiguración sindical en el Kirchnerismo. Revista Trabajo y Sociedad, 27, 23-41. Recuperado de http://www.unse.edu.ar/trabajoysociedad/27 \%20DOSSIER \%2003 \%20VENTRICI \%20DELFINI \%20Sindicalismo \%20argentino.pdf

Etchemendy, S., y Palermo, V. (1998). Conflicto y concertación. Gobierno, Congreso y organizaciones e interés en la reforma laboral del primer gobierno de Menem. Desarrollo Económico, 37(148), 559-590.

INDEC (2018). Informes Técnicos, vol. 1, № 63, cuarto trimestre de 2016.

Marticorena, C. (2015).Avances en el estudio de la relación sindicalismo y Kirchnerismo. Sociohistórica, 36, 1-22. Recuperado de http://www.memoria.fahce.unlp.edu.ar/art_revistas/pr.7052/pr.7052.pdf

Morgenstein, S. (2004). Patterns of Legislative Politics: Roll-Call Voting in Latin America and the United States, Nueva York: Cambridge University Press.

Natalucci, A., y Morris, M. B. (2016).La unidad de la CGT en perspectiva (2004-2016).Revista Socio Debates, 2(4), 33-62. Recuperado de http://www.feej.org/index.php/revista-sociodebate

Retamozo, M. y Morris, M. B. (2015). Sindicalismo y política: La Central de Trabajadores Argentinos en tiempos kirchneristas. Revista Estudios Sociológicos del Colegio de México, XXXIII(97),63-87. DOI https://10.24201/E S.2015V33N97.17

Sánchez Román, J. A. (2013).Los Argentinos y los impuestos. Buenos Aires: siglo veintiuno editores.

Schipiani, A. (2012). Los motivos de la fractura. Le monde Diplomatique, 57, Recuperado de https://www.eldiplo.or g/157-el-primer-opositor/los-motivos-de-la-fractura/ 
Tilly, Ch. (1998). Conflicto político y cambio social. En T. Ibarra y B. Tejerina (comps), Los movimientos sociales. Transformaciones políticas y cambio cultural (pp.25-41).Madrid: Trotta.

Zelaznik, J. (2015). Cómo construir mayorías en el Congreso. Recuperado de http://elestadista.com.ar/?p=8874

Zlotogwiazda, M. (23 de diciembre de 2016). Alergia a ganancias. El cronista. , Recuperado de https://www.cronista .com/columnistas/Alergia-a-Ganancias-20161223-0068.html

\section{Notas}

1 En julio de 2012 se produjo una ruptura de la CGT. La CGT Azopardo qudó bajo la conducción del líder camionero Hugo Moyano, y la CGT Alsina con la conducción de Antonio Caló, dirigente metalúrgico.

2 Esta agrupación se conformó en el 2008 bajo la conducción del dirigente gastronómico Luis Barrionuevo.

3 En el 2010 la CTA quedó dividida luego de un conflicto en las elecciones internas. La CTA de los Argentinos quedó liderada por Hugo Yasky, y la Autónoma por Pablo Micheli.

4 Cristina Fernández de Kirchner gobernó la Argentina durante dos mandatos consecutivos entre el 2007 y el 2015.

5 Subsecretaría de Políticas, Estadísticas y Estudios Laborales Ministerio de Trabajo, Empleo y Seguridad Social, 2017.

6 En el año 2016, según el Î́ndice de Precios al Consumidor del Congreso de la Nación, la inflación fue del 40 \% mientras para el INDEC fue del $30 \%$.

7 Los autores realizaron 20 entrevistas en profundidad a legisladores, dirigentes sindicales, funcionarios públicos, expertos en cuestiones fiscales, periodistas.

$8 \mathrm{El}$ análisis que aquí presentamos forma parte del proyecto "Estado, actores sociales y cuestión social: reconfiguración de las desigualdades y el conflicto social en la Argentina actual", financiado por el Consejo Nacional de Investigaciones Científicas y Técnicas- CONICET-, y la Universidad Nacional de General Sarmiento (2015-2019); y el proyecto "Desigualdades persistentes y nuevas desigualdades en la Argentina actual: procesos, escalas y dimensiones", Universidad Nacional de General Sarmiento (2014-2019).

9 Para un análisis de la ruptura de la CTA ver Retamozo y Morris, 2015;Armelino, 2012.

10 En tanto, la CGT Alsina y la CTA de los Argentinos, si bien en forma retórica principalmente y en reuniones con la presidenta se posicionaron a favor de revisar el IG, pero no acompañaron activamente ninguna medida de fuerza.

11 Medido en dólares el salario promedio registrado del sector privado aumentó entre el 2003 y el 2015 un 350\% (Herrera, H. http://eppa.com.ar/el-salario-en-dolares-en-la-argentina-una-evolucion-y-una-mirada-sobre-latinoamerica).

12 Mientras para el Instituto Nacional de Estadística y Censos -INDEC- la inflación anual desde el 2010 y hasta2015ha girado en torno al $10 \%$, para las consultoras privadas osciló entre un 20 y un $35 \%$.

13 https://docs.google.com/spreadsheets/d/1KM9oUktBCy2_SZrc6EemL4ytdoWh27bMcvfUL-JlStw/edit\#gid=0

$14 \mathrm{https} / /$ www.clarin.com/politica/sergio_massa-impuesto_a_las_ganancias-elecciones_2015-paro-frente_renovador_ 0_S1t0rbqPXx.html

15 https://www.politicargentina.com/notas/201510/8699-macri-prometio-eliminar-el-impuesto-a-las-ganancias-a-los-t rabajadores.html

16 Durante los meses de mayo y septiembre de 2016 se produjeron diversas protestas de extensión territorial impulsadas por ciudadanos, sector productivo, organizaciones sociales y políticas en reclamo por el aumento del gas.

17 Hasta entonces, según estimaciones de la AFIP, estaban alcanzados por el impuesto 1.300 .000 trabajadores.

18 Conocidos los anuncios, varios especialistas advirtieron que la mejora se diluiría tras la aplicación de las paritarias a lo largo del año.

19 La Ley Cerrojo o Ley 26017 se sancionó en el 2005 y establece que el Poder Ejecutivo no puede reabrir el proceso de canje de los bonos del Estado nacional. Asimismo, se prohíben las negociaciones con quienes no se incorporaron al canje a la fecha del 25 de febrero de 2005. La ley de pago soberano (26984), se aprobó en el 2014 en pleno conflicto del gobierno kirchnerista con los hold-outs y el juez norteamericano Thomas Griesa. La misma declaraba de interés público la reestructuración de la deuda soberana realizada en 2005 y 2010. La ley permitía el cobro a quienes tenían títulos públicos y se vieran impedidos de efectuarlo por órdenes judiciales dictadas por el mismo Griesa.

20 En este bloque se registraron las ausencias de Felipé Solá, Facundo Moyano y el líder cegetista Héctor Daer. Para fines de 2018 Solá y Moyano habían abandonado el espacio conducido por Sergio Massa para conformar el bloque Red por una Nueva Argentina.

21 Uno de los puntos clave de la aprobación de este amplio y heterogéneo proyecto fue la negociación entre el Poder Ejecutivo y las provincias por la cual se les devuelve a estas el $15 \%$ de la coparticipación que se les retenía para financiar la Anses. Si se tiene en cuenta que este capítulo de la ley fue aprobado por 62 votos a favor y solo 5 en contra, en este punto el gobierno encontró la llave para contar con el apoyo del peronismo encolumnado con los gobernadores. 
22 Jorge Taboada y Alberto Roberti (FR) fueron otros legisladores de origen sindical que acompañaron proyectos con cambios en el tributo.

23 Un aspecto conflictivo era la eliminación de la posibilidad de deducir del impuesto a cónyuges y padres a cargo, a la vez que se reducía de 24 a 18 años la edad de los hijos con posibilidad de deducirse MNI (La Nación, 23/11/2016).

24 Entre ellas, elevar el MNI al $60 \%$, nuevas deducciones (como hijo discapacitado, alquiler de vivienda única y zona desfavorable), y gravar la renta financiera para compensar los recursos que el Estado dejaría de recaudar.

25 La propuesta original del macrismo elevaba el MNI un $15 \%$, mientras que en el proyecto opositor el incremento era del $47 \%$. En el proyecto finalmente apoyado y convertido en ley, el aumento establecido fue del 22,8 \%.

26 Recordó el slogan "El Estado no tiene que quedarse con el fruto de tu trabajo. En mi gobierno los trabajadores no van a pagar el impuesto a las ganancias".

27 Las intervenciones de Alicia Siciliani por el Frente Progresista santafesino y de Oscar Romero por el bloque Justicialista también objetaron la escasa voluntad de consensuar el proyecto por parte del Poder Ejecutivo. Honorable Cámara de Diputados, versión taquigráfica del 6 de diciembre, pp. 141-143.

28 Los que acompañaron con su firma el proyecto opositor fueron los senadores Juan Manuel Abal Medina, Omar Perotti, Graciela de la Rosa, María Labado y Pedro Guastavino.

$29 \mathrm{Al}$ ser un impuesto coparticipable, los gobernadores adquirieron una importante presencia en las negociaciones parlamentarias.

30 Héctor Daer, Juan Carlos Schmid y Carlos Acuña encabezaron la CGT desde su reunificación en agosto de 2016.

31 La incorporación de los nuevos jueces al pago de ganancias fue otra modificación que contó con apoyo, aunque hubo varios legisladores que reclamaron que la medida se hiciera extensiva a los jueces actuales.

32 Los senadores Guillermo Pereyra (Movimiento Popular Neuquino), Luis Luenzo, María Elena Odarda (UNEN Río Negro), Juan Mario Pais y Daniel Llovera (FPV La Pampa) resaltaron estos cambios en sus respectivos votos a favor del proyecto aprobado en el Senado. Senado de la Nación, versión taquigráfica del 21 de diciembre, pp. 11, $25,27,32$ y 38.

33 Como Oscar Castillo/Frente Cívico y Social Catamarca, Dalmacio Mera PJ Catamarca, Carlos Caserio/PJ Córdoba, Juan Carlos Romero/PJ Salta, Jaime Linares/UNEN Provincia de Buenos Aires.

34 Diez abstenciones y los dos votos en contra fueron de senadores kirchneristas, mientras que las otras dos abstenciones correspondieron a los peronistas puntanos Adolfo Rodriguez Saá y Alicia Negre de Alonso

35 Alrededor de treinta mil pesos por entonces.

36 Las de feriado y días no laborales se deducen al $100 \%$. Ninguna de las horas extras puede hacer pasar de una escala a otra.

37 En tanto para los monotributistas, las escalas se incrementaron en un $75 \%$. Un aspecto vinculado a la equidad en el alcance del tributo fue la incorporación al gravamen de magistrados, funcionarios y empleados del Poder Judicial de la Nación, de las provincias y del Ministerio Público de la Nación que fueran nombrados a partir de 2017.

38 Los primeros \$ 20000 excedentes al año pagarían un $5 \%$ de ganancias; entre 20 y 40 mil, la tasa sube al $9 \%$, entre 40 y 60 mil, al $12 \%$ entre 60 y 80 mil al $15 \%$; entre 80 y 120 mil, al $19 \%$, entre 120 y 160 mil, al $23 \%$, entre 160 y 240 mil, al $27 \%$, entre 240 y 320 mil, al $31 \%$,y por encima de 320 mil, al $35 \%$. En el caso de los monotributistas, las escalas se elevaron en un $75 \%$ (Ambito Financiero, 23/12/2016).

39 Laspina, op. cit.

40 Si bien este legislador alertó que la experiencia del debate "nos dejó una enseñanza: no se gobierna con el látigo ni creyendo que somos los dueños absolutos de la verdad”, justificó la presencia de los diputados sanjuaninos en el recinto para manifestarse a favor de la quita de retenciones a la minería, tomar distancia y defenderse de las críticas de su propio bloque.

41 Según estimaciones proporcionadas por el gobierno la modificación del IG implicaba que más de un millón y medio de personas de clase alta y media-alta dejarán de pagar en 2017 \$49.598 millones, Dicho monto es equivalente al presupuesto que le asignaron al Ministerio de Salud para todo ese año, y este beneficio se sumaría a los $\$ 50.000$ millones que implicó la modificación del IG establecida para 2016. Así, los individuos que pertenecen a los dos deciles de ingresos más altos se ahorrarán al cabo de la finalización del ejercicio fiscal 2017 \$100.000 millones (Zlotogwiazda, El Cronista, 23/12/2016).

\section{BY-NC-SA}

